\title{
Alternative Technique for the Construction of Menarcheal Life-table
}

\author{
Tika Ram Aryal \\ Central Department of Statistics, Tribhuvan University, Kirtipur, Kathmandu \\ e-mail:traryal@rediffmail.com
}

\begin{abstract}
The main aim of this paper is to construct menarcheal life-table of Nepal using sample survey data. An alternative technique for the construction such as a life-table based on models has been proposed. The construction of such a life-table describes the distribution of the waiting time for getting menarche at birth as well as some specified age or age group of girls (in years). The average expected number of years to attain menarche at birth was found to be 14.8 years. Likewise, if girls who attain $12^{\text {th }}$ birthday, they most likely to wait another 3.2 years for getting menarche among older birth cohort girls while it was 2.4 years among younger birth cohort girls. Only $11 \%$ girls had attained menarche before their $13^{\text {th }}$ birthday. This finding may help for planners and policy-makers to design effective policy in reproductive health and behaviors of females in a country.
\end{abstract}

Key words: menarche, life-table, recall error, memory lapse, logistic distribution

\section{Introduction}

Menarche is one of the most important events among girls. It is most likely to be a recognizable event; however, that marked in many societies either by ritually and ceremonially (Aryal 2002). The onset of menarche is based on self-reported data of first bleeding experience among girls (Aryal 2004). This event is generally occurring at the age of 10 to 23 years both in developed and under-developed countries (Aryal 2005). Distributions of age at menarche show larger variation across the populations (Aryal 2002, Tiwari et al. 2005). Cohort studies are generally more reliable than cross-sectional or retrospective studies (Aryal 2007). The distributional pattern has been largely linkage but not completely broken with having an early menarche and with late marriage among western girls (Aryal 2008a). This fact has not necessarily proved by any single data set on the timing of menarche and on the relationship with marriage and the subsequent events at the individual level as documented by several researchers among predominantly and contemporary populations elsewhere (Aryal 2004, 2007, 2008a, Treloar 1974, Treloar et al. 1967).

Most of the women who had experienced menarche far earlier in their life and the data regarding the timing of menarche may have high chance of recall bias/ memory lapse and under and/or over reporting the events (Aryal 2002). The reporting bias or memory lapse of such data had a mean error of about 6 months and the errors were distributed randomly with respect to the age at menarche (Aryal 2005). The recall error may be a problem, especially if the recalled event was in the long distant past and the respondents were illiterate (Aryal 2002, 2008b). A number of studies comparing the actual or reporting or known age at menarche with self-report of age many years later have shown that recall errors can be quite large, though usually not biased along with have not any systematic trend (Damon et al. 1969, Livson \& McNeill 1962). However, the current status reporting research design data, one simply ascertains whether each girl at a given age has or has not experienced the first means, and then uses some statistical analysis to estimate means or medians of age of menarche in most of the populations (Finney 1971). Indeed, the cohort based study has provided the best distributional evidence for a secular decline in the age at menarche (Sandalar et al. 1984). The distribution indicated that 80 per cent of all United States girls start to menstruate between somewhere $11^{\text {th }}$ birthday until $14^{\text {th }}$ birthday, and with having median was little over 12 years (Aryal 2005). 
This suggests us that it is quite difficult to ascertain right age of menarche among girls due to huge memory bias/lapse for reporting the events. In such a situation, researchers developed models to know the right age of menarche, marriage and menopause among females and also to know the post-partum amenorrheal period among females (Aryal 2008b, 2009). Recently, Aryal (2005) applied probability model to describe the distribution of age at menarche among Nepalese girls. With the help of the fitted models, one can compute descriptive statistics of age at menarche in any populations. It is therefore this paper tries to develop an alternative technique to construct menarcheal lifetable with the help of the fitted model in order to know the expected waiting time for getting menarche at birth as well as to a specified age or age group of girls.

\section{Methodology}

This paper accomplishes the data from a sample survey entitled "Demographic Survey on Fertility and Mobility in Rural Nepal (DSFM 2000): A Study of Palpa and Rupandehi Districts” conducted between January and June 2000. A total of 811 households were surveyed. A total of 1258 girls had attained menarche on or before the survey date and they reported their age at menarche at the survey point. The details about survey are found in Aryal (2002, 2005). Further to examine the distributions of age at menarche of females, three birth cohorts such as the older birth cohort (birth before 1970), the intermediate birth cohort (birth between 1970 to 1979) and the younger birth cohort (birth on 1980 or later) were categorized for the construction of the menarcheal life-table.

\section{Construction of menarcheal life-table}

Aryal (2005) fitted logistic model to describe the distribution of age at menarche using retrospective reporting data of Nepal. The onset of menarche first increases with age, attains maximum in age range of 1415 years, and thereafter declines abruptly. Usually it starts in the interval of 10 years to 23 years. Taking this notion into account and other empirical findings, Aryal (2005) developed logistic model to describe the distribution of girls according to their age at menarche. The detail was found in Aryal (2005).

Now we introduce a concept to construct a menarcheal life-table through probability model. The procedure for constructing menarcheal life-table is also similar to that of the construction of mortality life-table. Since menarche is a universal event, and each and every girl would eventually have experienced it. Under this assumption, the expected waiting time for getting menarche in a certain age or age group is obtained by using life table technique with the help of the probability model. Number of girls who have had menarche in a certain age or age group was considered as the number of death cases as similar in the mortality life table. The proportions of surviving i.e. not having menarche in a specified age or age interval are obtained by fitting the logistic distribution. Once we get the proportions of surviving, i.e. proportions of not having menarche in a specified age or age group, the following procedure can be followed for the construction of menarcheal life-table.

Let $M_{x}$ be the proportion of females who were attaining the menarche at age $x$ years or less, and this value can be obtained by fitting logistic distribution. For construction of menarcheal life-table, we adopted $\mathrm{M}_{\mathrm{x}}$ values from Aryal (2005). $S_{x}$ be the proportion of surviving or not having menarche at age $x\left(S_{x}=1-M_{x}\right)$. If $l_{0}$ is the cohort of the life table, then $l_{x}=l_{0} * S_{x}$ and the probability of attaining menarche between $\mathrm{x}$ and $\mathrm{x}+1$ years of age is thus obtained by using following expression.

$$
q_{x}=\frac{\left(M_{x+1}-M_{x}\right)}{\left(1-M_{x}\right)}
$$

The other functions of the menarcheal life-table, $\mathrm{L}_{\mathrm{x}}$ (person-year attained menarche by the cohort at age $\mathrm{x}$ ), $\mathrm{T}_{\mathrm{x}}$ (total person-year attained menarcheal by the cohort after age $\mathrm{x}$ ) and $\mathrm{e}_{\mathrm{x}}$ (average number of years expected to attain menarche at age $\mathrm{x}$ ) are computed by the usual process followed in the construction of a mortality life-table. The computational expression of life-table functions are given below.

$$
\begin{aligned}
& \mathrm{L}_{\mathrm{x}}=\frac{\mathrm{n}\left(\mathrm{l}_{\mathrm{x}}+\mathrm{l}_{\mathrm{x}+n}\right)}{2}, \mathrm{~T}_{\mathrm{x}}=\sum_{x}^{n} \mathrm{~L}_{\mathrm{x}}+\mathrm{L}_{\mathrm{x}+1}+\ldots+\mathrm{L}_{\mathrm{x}+\mathrm{n}}, \\
& \mathrm{T}_{\mathrm{x}+1}=\mathrm{T}_{\mathrm{x}}-\mathrm{L}_{\mathrm{x}} \text { and } \mathrm{e}_{\mathrm{x}}=\frac{\mathrm{T}_{\mathrm{x}}}{\mathrm{l}_{\mathrm{x}}}
\end{aligned}
$$

\section{Results and Discussion}

The results of constructed menarcheal life-table of different birth cohorts are presented in the following tables. 
Tika Ram Aryal /Alternative Technique.

Table 1. Menarcheal life-table (for older birth cohort, i.e. birth before 1970)

\begin{tabular}{l|l|l|l|l|l|l|l}
\hline Age at menarche & $\mathbf{M}_{\mathbf{x}}$ & $\mathbf{S}_{\mathbf{x}}$ & $\mathbf{l}_{\mathbf{x}}$ & $\mathbf{q}_{\mathbf{x}}$ & $\mathbf{L}_{\mathbf{x}}$ & $\mathbf{T}_{\mathbf{x}}$ & $\mathbf{e}_{\mathbf{x}}$ \\
\hline $0-12$ & 0.00621 & 1.00000 & 100000.00 & 0.00621 & 1196276.95 & 1513290.24 & 15.13 \\
$12-13$ & 0.10987 & 0.99379 & 99379.49 & 0.10431 & 94196.39 & 317013.28 & 3.19 \\
$13-14$ & 0.25023 & 0.89013 & 89013.28 & 0.15768 & 81995.36 & 222816.90 & 2.50 \\
$14-15$ & 0.47434 & 0.74977 & 74977.45 & 0.29891 & 63771.61 & 140821.53 & 1.88 \\
$15-16$ & 0.70930 & 0.52566 & 52565.78 & 0.44697 & 40818.10 & 77049.92 & 1.47 \\
$16-17$ & 0.86837 & 0.29070 & 29070.41 & 0.54722 & 21116.52 & 36231.82 & 1.25 \\
$17-18$ & 0.94692 & 0.13163 & 13162.63 & 0.59671 & 9235.49 & 15115.30 & 1.15 \\
$18-19$ & 0.97969 & 0.05308 & 5308.36 & 0.61736 & 3669.77 & 5879.81 & 1.11 \\
$19-20$ & 0.99239 & 0.02031 & 2031.18 & 0.62537 & 1396.06 & 2210.04 & 1.09 \\
$20-21$ & 0.99717 & 0.00761 & 760.95 & 0.62838 & 521.87 & 813.98 & 1.07 \\
$21-22$ & 0.99895 & 0.00283 & 282.78 & 0.62950 & 193.78 & 292.11 & 1.03 \\
$22-23$ & 0.99961 & 0.00105 & 104.77 & 0.62992 & 71.77 & 98.33 & 0.94 \\
$23-24$ & 0.99986 & 0.00039 & 38.77 & 0.63007 & 26.56 & 26.56 & 0.68 \\
\hline
\end{tabular}

$M_{x}$ value was taken from Aryal(2005)

Menarcheal life-table has been constructed by taking the value of $\mathrm{M}_{\mathrm{x}}$ from fitted logistic model of different birth cohorts of Nepalese females. Figures in column 2 $\left(\mathrm{M}_{\mathrm{x}}\right)$ of Table 1 are based on the proportions of females who attain menarche for a particular age $\mathrm{x}$ among older birth cohort females i.e. birth before 1970. The menarcheal life-table suggested that the average expected number of years to attain menarche at birth was 15.1 years for the data of older birth cohort females, which are also consistent findings of the median age at menarche (Aryal 2002). Like-wise, the menarcheal life-table provides the remaining waiting number of years for attaining menarche at a specified age of females. For instance, the expected waiting time was found to be 3.2, 1.3 and 1.1 years for females who reached their $12^{\text {th }}, 16^{\text {th }}$ and $20^{\text {th }}$ birthdays respectively. It is also observed that about $11,47,87$ and 98 per cent females attain their menarche before they are reaching their $13^{\text {th }}, 15^{\text {th }}, 17^{\text {th }}$ and $19^{\text {th }}$ birthdays respectively.

Table 2. Menarcheal life-table (for intermediate birth cohort i.e. birth between 1970-1979)

\begin{tabular}{l|l|l|l|l|l|l|l}
\hline Age at menarche & $\mathbf{M}_{\mathbf{x}}$ & $\mathbf{S}_{\mathbf{x}}$ & $\mathbf{l}_{\mathbf{x}}$ & $\mathbf{q}_{\mathbf{x}}$ & $\mathbf{L}_{\mathbf{x}}$ & $\mathbf{T}_{\mathbf{x}}$ & $\mathbf{e}_{\mathbf{x}}$ \\
\hline $0-12$ & 0.00521 & 1.00000 & 100000.00 & 0.00521 & 1196872.19 & 1486742.84 & 14.87 \\
$12-13$ & 0.11999 & 0.99479 & 99478.70 & 0.11538 & 93739.63 & 289870.65 & 2.91 \\
$13-14$ & 0.28778 & 0.88001 & 88000.56 & 0.19066 & 79611.25 & 196131.02 & 2.23 \\
$14-15$ & 0.54491 & 0.71222 & 71221.94 & 0.36102 & 58365.69 & 116519.78 & 1.64 \\
$15-16$ & 0.78013 & 0.45509 & 45509.44 & 0.51686 & 33748.40 & 58154.09 & 1.28 \\
$16-17$ & 0.91315 & 0.21987 & 21987.36 & 0.60499 & 15336.26 & 24405.69 & 1.11 \\
$17-18$ & 0.96890 & 0.08685 & 8685.15 & 0.64193 & 5897.52 & 9069.43 & 1.04 \\
$18-19$ & 0.98928 & 0.03110 & 3109.88 & 0.65544 & 2090.72 & 3171.91 & 1.02 \\
$19-20$ & 0.99636 & 0.01072 & 1071.55 & 0.66012 & 717.87 & 1081.20 & 1.01 \\
$20-21$ & 0.99877 & 0.00364 & 364.20 & 0.66172 & 243.70 & 363.32 & 1.00 \\
$21-22$ & 0.99958 & 0.00123 & 123.20 & 0.66226 & 82.41 & 119.63 & 0.97 \\
$22-23$ & 0.99986 & 0.00042 & 41.61 & 0.66244 & 27.83 & 37.22 & 0.89 \\
$23-24$ & 0.99995 & 0.00014 & 14.05 & 0.66250 & 9.39 & 9.39 & 0.67 \\
\hline \multicolumn{7}{c}{$M_{x}$ value was taken from Aryal(2005) } \\
\hline
\end{tabular}

Menarcheal life-table for the data of intermediate birth cohort females has been constructed and presented in Table 2. Menarcheal life-table suggested that the average expected number of years to attain menarche at birth was found to be 14.9 years for intermediate birth cohort, i.e. birth between 1970-1979, which is consistent estimate of the median age at menarche obtained by computing actual data (Aryal 2002). This life-table also provides the remaining waiting time for getting menarche for a particular age or age group of 
females. The expected remaining waiting time was found to be 2.9, 1.1, and 1.0 years for those females who reached their $12^{\text {th }}, 16^{\text {th }}$ and $20^{\text {th }}$ birthdays respectively. The menarcheal life-table also revealed that around 12, 54, 91 and 99 per cent females who attain their menarche before they are reaching their $13^{\text {th }}, 15^{\text {th }}, 17^{\text {th }}$ and $19^{\text {th }}$ birthdays respectively.

Table 3. Menarcheal life-table (for younger birth cohort, i.e. birth on 1980 and later)

\begin{tabular}{l|l|l|l|llll}
\hline Age at menarche & $\mathbf{M}_{\mathbf{x}}$ & $\mathbf{S}_{\mathbf{x}}$ & $\mathbf{l}_{\mathbf{x}}$ & $\mathbf{q}_{\mathbf{x}}$ & $\mathbf{L}_{\mathbf{x}}$ & $\mathbf{T}_{\mathbf{x}}$ & $\mathbf{e}_{\mathbf{x}}$ \\
\hline $0-12$ & 0.00480 & 1.00000 & 100000.00 & 0.00480 & 1197119.64 & 1437938.84 & 14.38 \\
$12-13$ & 0.16221 & 0.99520 & 99519.94 & 0.15817 & 91649.60 & 240819.20 & 2.42 \\
$13-14$ & 0.39864 & 0.83779 & 83779.27 & 0.28221 & 71957.53 & 149169.60 & 1.78 \\
$14-15$ & 0.69416 & 0.60136 & 60135.79 & 0.49142 & 45359.87 & 77212.07 & 1.28 \\
$15-16$ & 0.88599 & 0.30584 & 30583.94 & 0.62722 & 20992.51 & 31852.20 & 1.04 \\
$16-17$ & 0.96378 & 0.11401 & 11401.07 & 0.68229 & 7511.66 & 10859.70 & 0.95 \\
$17-18$ & 0.98914 & 0.03622 & 3622.24 & 0.70025 & 2354.01 & 3348.04 & 0.92 \\
$18-19$ & 0.99680 & 0.01086 & 1085.78 & 0.70567 & 702.68 & 994.02 & 0.92 \\
$19-20$ & 0.99906 & 0.00320 & 319.58 & 0.70727 & 206.56 & 291.34 & 0.91 \\
$20-21$ & 0.99973 & 0.00094 & 93.55 & 0.70774 & 60.45 & 84.78 & 0.91 \\
$21-22$ & 0.99992 & 0.00027 & 27.34 & 0.70788 & 17.66 & 24.33 & 0.89 \\
$22-23$ & 0.99998 & 0.00008 & 7.99 & 0.70792 & 5.16 & 6.67 & 0.83 \\
$23-24$ & 0.99999 & 0.00002 & 2.33 & 0.70793 & 1.51 & 1.51 & 0.65 \\
\hline \multicolumn{7}{c}{$M_{x}$ value was taken from Aryal(2005) } \\
\hline
\end{tabular}

waiting time at birth is somewhat consistent with the median age at menarche (Aryal 2002). The expected waiting time for getting menarche was found to be 2.4 , 1.0 and 0.9 years for those females who reached their $12^{\text {th }}, 16^{\text {th }}$, and $20^{\text {th }}$ birthdays respectively. Over 16,69 , 96 and 100 per cent females who attain their menarche before they are reaching their $13^{\text {th }}, 15^{\text {th }}, 17^{\text {th }}$ and $19^{\text {th }}$ birthdays respectively.

Table 4. Menarcheal life-table (for all females)

\begin{tabular}{|c|c|c|c|c|c|c|c|}
\hline Age at menarche & $\mathbf{M}_{\mathbf{x}}$ & $S_{x}$ & $l_{x}$ & $\mathbf{q}_{\mathrm{x}}$ & $\mathbf{L}_{x}$ & $\mathbf{T}_{\mathrm{x}}$ & $\mathbf{e}_{\mathrm{x}}$ \\
\hline $0-12$ & 0.00853 & 1.00000 & 100000.00 & 0.00853 & 1194880.63 & 1474525.53 & 14.75 \\
\hline $12-13$ & 0.15160 & 0.99147 & 99146.77 & 0.14430 & 91993.24 & 279644.91 & 2.82 \\
\hline $13-14$ & 0.32938 & 0.84840 & 84839.71 & 0.20954 & 75950.99 & 187651.67 & 2.21 \\
\hline $14-15$ & 0.57446 & 0.67062 & 67062.27 & 0.36546 & 54808.04 & 111700.68 & 1.67 \\
\hline $15-16$ & 0.78771 & 0.42554 & 42553.82 & 0.50112 & 31891.55 & 56892.64 & 1.34 \\
\hline $16-17$ & 0.91070 & 0.21229 & 21229.27 & 0.57937 & 15079.52 & 25001.09 & 1.18 \\
\hline $17-18$ & 0.96555 & 0.08930 & 8929.76 & 0.61426 & 6187.16 & 9921.57 & 1.11 \\
\hline $18-19$ & 0.98719 & 0.03445 & 3444.55 & 0.62802 & 2362.92 & 3734.42 & 1.08 \\
\hline $19-20$ & 0.99530 & 0.01281 & 1281.29 & 0.63318 & 875.65 & 1371.50 & 1.07 \\
\hline $20-21$ & 0.99828 & 0.00470 & 470.00 & 0.63508 & 320.75 & 495.85 & 1.06 \\
\hline $21-22$ & 0.99938 & 0.00172 & 171.51 & 0.63578 & 116.99 & 175.10 & 1.02 \\
\hline $22-23$ & 0.99977 & 0.00062 & 62.47 & 0.63603 & 42.60 & 58.11 & 0.93 \\
\hline $23-24$ & 0.99992 & 0.00023 & 22.74 & 0.63612 & 15.50 & 15.50 & 0.68 \\
\hline
\end{tabular}

The life-table provides that the average expected number of years to attain menarche at birth was found to be 14.8 years for data consisting all females (Table 4). The expected waiting number of years was found to be $2.8,1.2$ and 1.0 years at age of 12,16 and 20 years respectively. The life-table also reveals that about 15 ,
57, 91 and 99 per cent females who attain menarche before they are reaching $13^{\text {th }}, 15^{\text {th }}, 17^{\text {th }}$ and $19^{\text {th }}$ birthdays respectively. Since expected waiting time at birth was 14.8 years computed by life-table technique, which is consistent estimate of median age at menarche of 14.7 years computed by actual data sets (Aryal 2002). 
Finally, over 11, 12 and 16 per cent females are attaining their menarcheal age before they are reaching $13^{\text {th }}$ birthday for older birth cohort, intermediate birth cohort and younger birth cohort respectively. Similarly over 47, 54 and 69 per cent females are attaining menarche before reaching their $15^{\text {th }}$ birthdays for older birth cohort, intermediate birth cohort and younger birth cohort respectively. Like-wise over 87, 91 and 96 per cent females are attaining menarche before reaching their $17^{\text {th }}$ birthday for older birth cohort, intermediate birth cohort and younger birth cohort respectively. Over 98, 99 and 100 per cent females who are attaining menarche before they are reaching their $19^{\text {th }}$ birthday for the data of older, intermediate and younger birth cohort respectively. Thus these evidences have shown the distributional pattern of age at menarche over the generation of Nepalese females.

This paper uses alternative technique to compute the expected waiting time for getting married among Nepalese girls through construction of menarcheal life-table. With the help of the fitted model, we are constructed menarche life-table, which describes the distribution of the waiting time for getting menarche at birth as well as some specified age or age group of females that provides a precise explanation of age at menarche among Nepalese females. It was found that the average expected number of years to attain menarche at birth was 14.8 years for all girls whereas it was 15.2 years for older birth cohort (birth before 1970) and 14.4 years for younger birth cohort (birth on 1980 and later).

Similarly, expected waiting time for attaining menarche for a particular age or age group of girls was also obtained. For instance, if girls who attain $12^{\text {th }}$ birthday, they most likely to wait for getting menarche for another 3.2 years among girls of older birth cohort while it was 2.4 years among girls of younger birth cohort. Only 11 per cent girls had attained menarche before they are reaching $13^{\text {th }}$ birthday among girls of older birth cohort (birth before 1970) while 12 per cent and 16 per cent girls who attained menarche before they are reaching $13^{\text {th }}$ birthday among girls of intermediate and younger birth cohort respectively. This finding may help for planners and policy-makers to design effective policy in reproductive health and behaviors of females in a country.

\section{References}

Apter, D. and R. Vihko. 1983. Early menarche, a risk factor for breast cancer, indicates early onset of ovulatory cycles. Journal of Clinical Endocrinology and Metabolism 57:82-86.

Aryal, T.R. 2002. Some demographic models and their applications with reference to Nepal. $\mathrm{PhD}$ thesis, Department of Statistics, BHU, India.

Aryal, T.R. 2004. Age at menarche: differentials and determinants. Journal of Nepal Medical Association 43(152):71-75.

Aryal, T.R. 2005. A probability model for describing the distribution of age at menarche. Nepal Journal of Science and Technology 6:47-52.

Aryal, T.R. 2007. Age at first marriage in Nepal: differentials and determinants. Journal of Biosocial Science 39 (part 5):693-706.

Aryal, T.R. 2008a. Mathematical model for describing the distribution of female age at marriage in Nepal. Nepali Mathematical Sciences Report 28(1\&2):21-30.

Aryal, T.R. 2008b. An indirect technique to estimate the duration of post-partum amenorrhea. Nepal Journal of Science and Technology 8:137-142.

Aryal, T.R. 2009. Estimation of the duration of post-partum amenorrhea among Nepalese mothers: some indirect techniques. Nepal Journal of Science and Technology 10:137-142.

Damon, A., S.T. Damon, R.B. Reed and I. Valadian. 1969. Age at menarche of mothers and daughters, with a note on accuracy of recall. Human Biology 41:161175.

Finney, D.J. 1971. Statistical method in biological assay. Second edition, London: Griffin.

Foster, D.L., S.M. Yen and D.H. Osler. 1985. Internal and external determinants of the timing of puberty in the female. Journal of Reproduction and Fertility 75:327-344.

Johnson, J.L. and S. Kotz. 1970. Continuous univariate distributions-1. John Wiley and Sons, New York, USA.

Livson, N. and D. McNeill. 1962. The accuracy of recalled age at menarche. Human Biology 34:218-221.

Sandler, D.P., A.J. Wilcox and L.F. Horney. 1984. Age at menarche and subsequent reproductive events. American Journal of Epidemiology 119:765-774.

Treloar, A.E. 1974. Menarche, menopause and intervening fertility. Human Biology 46:485.

Treloar, A.E., R.E. Boynton, B.G. Behn and B.W. Brown. 1967. Variation of the human menstrual cycle through reproductive life. International Journal of Fertility 12:77-126.

Wood, J.W. 1992. Fertility and reproductive biology in Papua New Guinea. In: Human biology in Papua New Guinea: The small cosmos (Eds. R.D. Attenborough and M.P. Alpers ) Clarendon Press, Oxford, UK. 
Nepal Journal of Science and Technology 11 (2010) 199-204 